\title{
Acoustic Reverse-time Migration using Optimal Staggered-grid Finite-difference Operator Based on Least Squares
}

\author{
Hongyong YAN ${ }^{1}$, Lei YANG ${ }^{1,2}$, and Hong LIU $^{1}$ \\ ${ }^{1}$ Key Laboratory of Petroleum Resources Research, Institute of Geology \\ and Geophysics, Chinese Academy of Sciences, Beijing, China; \\ e-mail: yanhongyong@163.com \\ ${ }^{2}$ University of Chinese Academy of Sciences, Beijing, China
}

\begin{abstract}
Reverse-time migration (RTM) directly solves the two-way wave equation for wavefield propagation; therefore, how to solve the wave equation accurately and quickly is very important for RTM. The conventional staggered-grid finite-difference (SFD) operators are usually based on the Taylor-series expansion theory. If they are used to solve wave equation on a larger frequency content, a strong dispersion will occur, which directly affects the seismic image quality. In this paper, we propose an optimal SFD operator based on least squares to solve acoustic wave equation for prestack RTM, and obtain a new antidispersion RTM algorithm that can use short spatial difference operators. The synthetic and real data tests demonstrate that the least squares SFD (LSSFD) operator can mitigate the numerical dispersion, and the acoustic RTM using the LSSFD operator can effectively improve image quality comparing with that using the Taylor-series expansion SFD (TESFD) operator. Moreover, the LSSFD method can adopt a shorter spatial difference operator to reduce the computing cost.
\end{abstract}

Key words: migration; difference operator; image quality; least squares.

Ownership: Institute of Geophysics, Polish Academy of Sciences;

(C) 2015 Yan et al. This is an open access article distributed under the Creative Commons Attribution-NonCommercial-NoDerivs license,

http://creativecommons.org/licenses/by-nc-nd/3.0/. 


\section{INTRODUCTION}

Reverse-time migration (RTM) provides a superior way to image steeply dipping reflectors and complex subsurface structures in seismic exploration, because it is based on computing numerical solutions to a two-way wave equation (Baysal et al. 1983, McMechan 1983, Zhang and Zhang 2009, Pestana et al. 2012). The wave equation solutions are to describe the wavefield propagation features, and accurately image the subsurface structure with the wavefield information (Yan et al. 2013). The accuracy and efficiency of the RTM is strongly dependent on the algorithms used for numerical solutions of wave equation (Pestana and Stoffa 2009, Tessmer 2011). Therefore, how to solve the wave equation accurately and quickly is very important in RTM. At present, some ways have been used to solve wave equation, such as finite-difference (FD) methods, finite-element methods, and pseudo-spectral methods. But one of the most popular and easiest ways to implement numerical modeling and RTM based on the numerical solutions of wave equation is to use FD methods, including explicit methods and implicit methods, which mainly contain regular grid FD (Liu et al. 1998, Kosloff et al. 2010, Yan and Liu 2013) and staggered-grid FD (Virieux 1986, Pei 2004). These FD methods are flexible and efficient (Li et al. 2013). Furthermore, the staggered-grid FD methods have better accuracy and stability compared to the regular grid FD methods (Igel et al. 1992).

However, the numerical dispersion is an unavoidable weakness for FD methods (Pestana and Stoffa 2010), and it directly affects the image quality (Liu et al. 2008). To achieve higher accuracy and less dispersion, staggeredgrid finite-difference (SFD) methods require higher-order SFD operators to compute the spatial derivatives, and the advantages of high-order SFD operators in solving the wave equation have been presented by many scholars (Dong et al. 2000, Pei 2004). The conventional classic coefficients of the higher-order SFD operator on spatial derivatives are usually determined by a Taylor-series expansion of spatial derivative term (Dong et al. 2000). If these difference operators are directly chosen to compute spatial derivatives on a relatively wider frequency band in solving the wave equation, a strong dispersion will still occur. To effectively solve this problem, some optimization methods for the coefficients of SFD operators have been proposed to improve the accuracy and efficiency for modeling and imaging, such as Newton method (Kindelan et al. 1990), implicit scheme (Liu and Sen 2009), time-space domain dispersion-relation-based method (Liu and Sen 2011), scaled binomial windows (Chu and Stoffa 2012), and simulated annealing algorithm (Zhang and Yao 2013). However, most of the optimization methods are too complicated to become widely implemented in seismic imaging. Liu (2013) proposed an easy way to obtain optimal finite-difference operator 
based on least squares on a wide frequency zone, but his optimal finitedifference operator only applied to the second-order spatial derivatives for the regular grid. Yang et al. (2014) derived the difference coefficients for the first-order spatial derivatives by the dispersion relation and the least squares theory; however, these difference coefficients were only used for elastic modeling, and were not further extended to seismic imaging.

In this paper, we propose an optimal SFD operator based on least squares to perform wavefield forward and backward extrapolations for acoustic prestack RTM. We first introduce the least squares staggered-grid finitedifference (LSSFD) operator for solving the acoustic wave equation, then test the LSSFD operator by acoustic RTM with the synthetic and real data. Meanwhile, the numerical dispersion, modeling and imaging accuracy, and their computational efficiency are analyzed. Moreover, we compare the LSSFD operator with the conventional classic SFD operator based on Taylor-series expansion in the test application.

\section{THEORY AND METHOD}

\subsection{Optimal staggered-grid finite-difference operator based on least squares}

In this section, we introduce the optimal staggered-grid finite-difference operator and illustrate its basic formulation. We discuss the acoustic isotropic case, which is described by the 2D acoustic wave equation (Claerbout 1985)

$$
\frac{\partial}{\partial x}\left(\frac{1}{\rho} \frac{\partial p}{\partial x}\right)+\frac{\partial}{\partial z}\left(\frac{1}{\rho} \frac{\partial p}{\partial z}\right)=\frac{1}{\rho v^{2}} \frac{\partial^{2} p}{\partial t^{2}}
$$

where $x$ and $z$ are the space coordinates, $\rho$ is the density, $v$ is the velocity, $p$ represents the pressure, and $t$ is the time.

In order to solve Eq. 1, the temporal derivative is usually calculated by the second-order finite-difference scheme, and the spatial derivatives are calculated by the high-order SFD schemes to improve the accuracy. The $(2 M)$ th-order SFD schemes for the first-order spatial derivative of the pressure $p(x, z)$ can be expressed as follows (Kindelan et al. 1990):

$$
\begin{aligned}
& \frac{\partial p}{\partial x} \approx \frac{\delta p}{\delta x}=\frac{1}{\Delta x} \sum_{m=1}^{M} c_{m}[p(x+m \Delta x-0.5 \Delta x, z)-p(x-m \Delta x+0.5 \Delta x, z)], \\
& \frac{\partial p}{\partial z} \approx \frac{\delta p}{\delta z}=\frac{1}{\Delta z} \sum_{m=1}^{M} c_{m}[p(x, z+m \Delta z-0.5 \Delta z)-p(x, z-m \Delta z+0.5 \Delta z)],
\end{aligned}
$$

where $\Delta x$ and $\Delta z$ are the grid intervals, and $c_{m}(m=1,2, . ., M)$ are the SFD coefficients on spatial derivatives. Conventional classic SFD operator derives spatial high-order difference coefficients $c_{m}$ by the Taylor-series ex- 
pansion (Dong et al. 2000), so we call it Taylor-series expansion staggeredgrid finite-difference (TESFD) when using these spatial difference coefficients to solve wave equations. In this paper, we adopt the least squares method proposed by Yang et al. (2014) to determine the optimal coefficients of high-order SFD operators on spatial derivatives.

We take the spatial derivative about the $x$ direction as an example. Using the plane wave theory, we let

$$
p(x+m \Delta x)=p_{0} e^{i k(x+m \Delta x)},
$$

where $p_{0}$ is a constant, $i=\sqrt{-1}$, and $k$ is the wavenumber. Substituting Eq. 3 into Eq. 2a, and simplifying it, we obtain

$$
\beta \approx \sum_{m=1}^{M} c_{m} \sin [(2 m-1) \beta],
$$

where $\beta=k h / 2, h$ is the grid size, $h=\Delta x$ and $0 \leq \beta \leq \pi / 2$.

Because the left-hand side of Eq. 4 is the reserved item of the spatial derivative, and the right-hand side is the reserved item of the SFD schemes, we can construct a square error function based on dispersion relation (Yang et al. 2014)

$$
E\left(c_{1}, c_{2}, \cdots c_{M}\right)=\int_{0}^{b}\left\{\beta-\sum_{m=1}^{M} c_{m} \sin [(2 m-1) \beta]\right\}^{2} d \beta,
$$

where $b$ is the upper limit of the integral. The quadratic function 5 represents the square error introduced by the SFD schemes over the given interval $[0, b]$ in solving spatial derivative, and we determine its minimum value with the least squares theory (Yang et al. 2014). According to the necessary condition of extremum, we get

$$
\frac{\partial E\left(c_{1}, c_{2}, \cdots c_{M}\right)}{\partial c_{m}}=0
$$

Substituting Eq. 5 into Eq. 6, we can obtain the optimal coefficients of high-order SFD operators by solving the following equations (see the Appendix A for more details):

$$
\begin{array}{r}
\int_{0}^{b} \beta \sin [(2 n-1) \beta] d \beta-\sum_{m=1}^{M} c_{m}\left\{\int_{0}^{b} \sin [(2 n-1) \beta] \times \sin [(2 m-1) \beta] d \beta\right\}=0, \\
(n=1,2,3, \ldots, M) .
\end{array}
$$




\subsection{The basic workflow of acoustic RTM}

The acoustic prestack RTM used has three main parts: forward extrapolating the wavefield from the source, backward propagating the wavefield by reducing time from the recorded seismic data, and applying a proper imaging condition to construct an image where the reflection occurred (Zhang et al. 2010, Liu et al. 2011, Yan et al. 2013). In practice, the most important part of RTM is solving the wave equation for the forward and backward wavefields. We adopt the LSSFD operator presented in the previous section to solve the acoustic wave equation to improve the imaging accuracy for RTM. Moreover, there are some classes of imaging conditions that can be used to produce an image of the subsurface by propagating wavefields (Chattopadhyay and McMechan 2008). Here, we choose the source-normalized cross-correlation imaging condition (Leveille et al. 2011):

$$
I(x, z)=\frac{\int s(x, z, t) r(x, z, t) d t}{\int|s(x, z, t)|^{2} d t+\sigma},
$$

where $I(x, z)$ is the migration result of the position $(x, z), s(x, z, t)$ is the forward extrapolated wavefield form the source, $r(x, z, t)$ is the backward propagated wavefield from the recorded seismic data, and $\sigma$ is a small undefined and arbitrary damping factor.

Unfortunately, the imaging condition defined in Eq. 8 usually generates the low frequency noises (Liu et al. 2011). In this paper, we apply a Laplacian filter presented by Zhang and Sun (2009) to suppress the low frequency noises.

\section{NUMERICAL RESULTS}

\subsection{Numerical dispersion analysis}

According to Eq. 4, we define $\delta(\beta)$ as follows to describe the numerical dispersion of SFD:

$$
\delta(\beta)=\frac{\sum_{m=1}^{M} c_{m} \sin [(2 m-1) \beta]}{\beta}-1 .
$$

If $\delta(\beta)=0$, there is no numerical dispersion. If $\delta(\beta)$ is far from 0 , a large numerical dispersion will occur. Figure 1 shows variations of $\delta(\beta)$ with $\beta$ for different $M$ by the TESFD and LSSFD of relative error of numerical dispersion relation, respectively. From Fig. 1, we can see that the LSSFD operator widens the frequency zone for the same dispersion and the same spatial difference operator length parameter $M$, compared to the TESFD operator. 


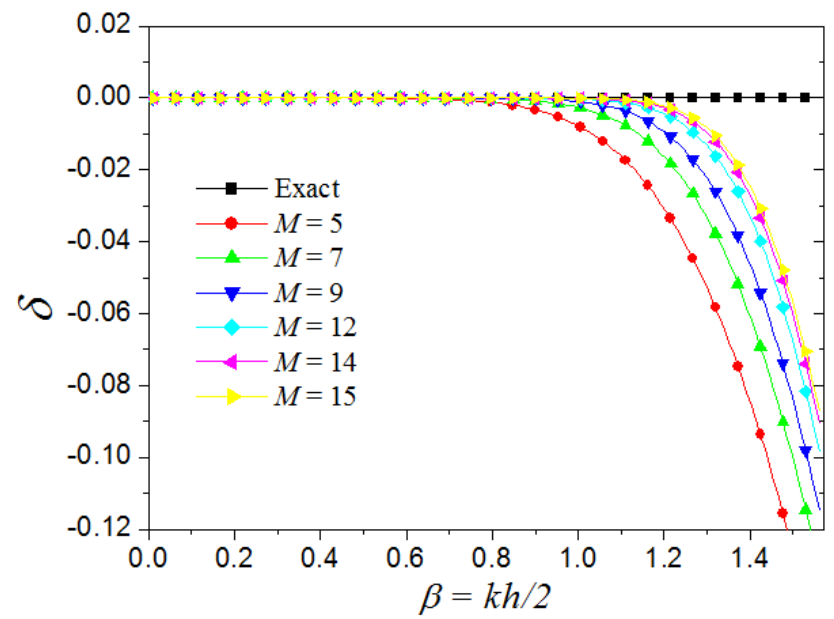

(a)

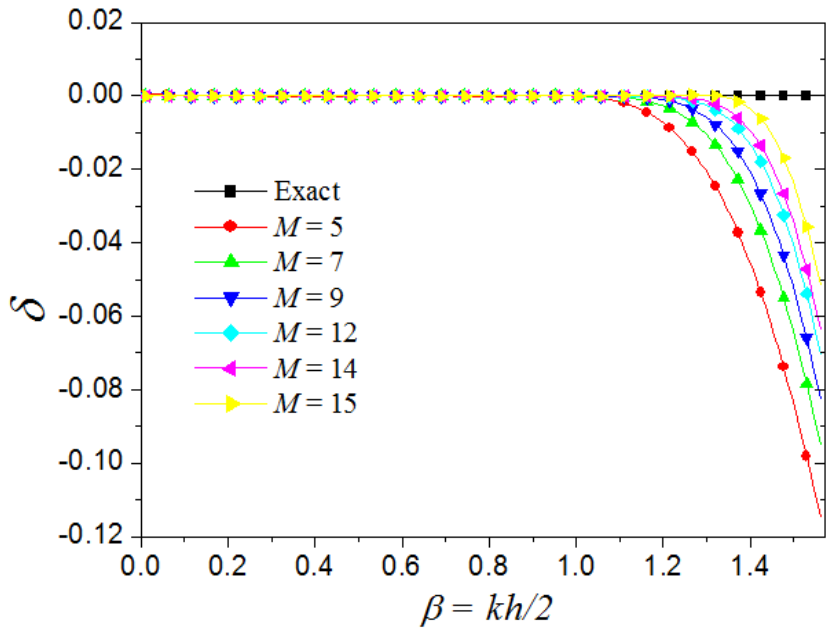

(b)

Fig. 1. Variations of $\delta(\beta)$ with $\beta$ for different $M$ by the TESFD operators and the LSSFD operators of a relative error of numerical dispersion relation, respectively: (a) by the TESFD, and (b) by the LSSFD.

From the comparison, the accuracy of the 10th-order LSSFD operator is much higher than that of the 10th-order TESFD operator, and even reaches that of the 18th-order TESFD operator because their relative error curves share almost the same position in Fig. 1. Namely, the accuracy of the LSSFD operators is much higher than that of the TESFD operators when using the same spatial difference operator length. Additionally, the length of LSSFD operator is shorter than that of TESFD operator under the same numerical accuracy. 


\subsection{Numerical modeling and RTM for fault model}

We use a fault model with two horizontal and one dipping interfaces shown in Fig. 2 to demonstrate effects of numerical modeling and RTM. The model is discretized into 401 by 201 grid points, with a grid interval of $10 \mathrm{~m}$. The velocity and the density for the first layer are $1600 \mathrm{~m} / \mathrm{s}$ and $1700 \mathrm{~kg}^{3} \mathrm{~m}^{-3}$, respectively, and for the second layer they are $2000 \mathrm{~m} / \mathrm{s}$ and $1800 \mathrm{~kg}^{3} \mathrm{~m}^{-3}$, respectively. We perform numerical modeling and prestack RTM using the SFD methods of 2nd-order accuracy in time and high-order accuracy in space. The source function is a Ricker wavelet with a peak frequency of $30 \mathrm{~Hz}$. There are 73 sources in total, and the sources are placed at a horizontal interval of $50 \mathrm{~m}$; the first source is located at $(100 \mathrm{~m}, 90 \mathrm{~m})$. Each source has 400 receivers, with a receiver interval of $10 \mathrm{~m}$. The time step is $0.001 \mathrm{~s}$ with $3 \mathrm{~s}$ total propagation time.

Figure 3 shows the modeling acoustic seismograms for the fault model computed by the LSSFD and the TESFD operators, respectively, when the source is located at $(2000 \mathrm{~m}, 60 \mathrm{~m})$. Figure 4 is the records extracted from Fig. 3. Figure 5 shows the corresponding snapshots of the seismograms in Fig. 3 at $t=1 \mathrm{~s}$. From Figs. 3, 4, and 5, we observe that the waveforms on both seismograms and snapshots computed by the 8th-order LSSFD operator and the 16th-order TESFD operator have very small numerical dispersion. However, the waveforms on both seismogram and snapshot computed by the 8th-order TESFD operator have obvious numerical dispersion and waveform distortion. By comparing these seismograms and snapshots, we can find that the acoustic modeling accuracy by the 8th-order LSSFD operator is much higher than that by the 8th-order TESFD operator, and even slightly higher than that by the 16th-order TESFD operator. Note that these accuracy analyses based on acoustic modeling are fully consistent with the theoretical analyses in the previous section.

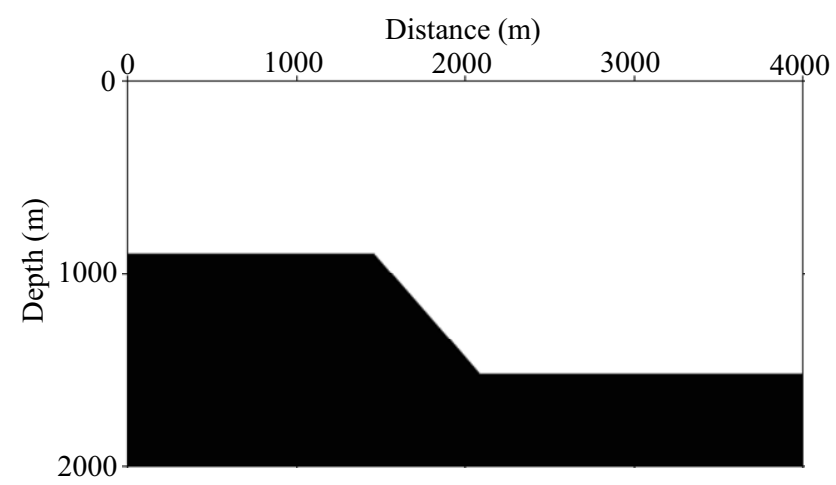

Fig. 2. The fault model. 


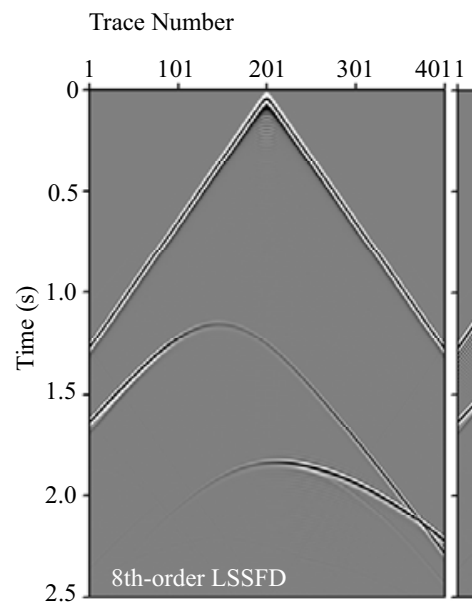

(a)

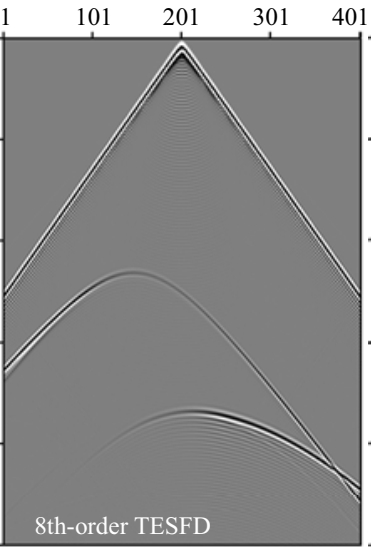

(b)

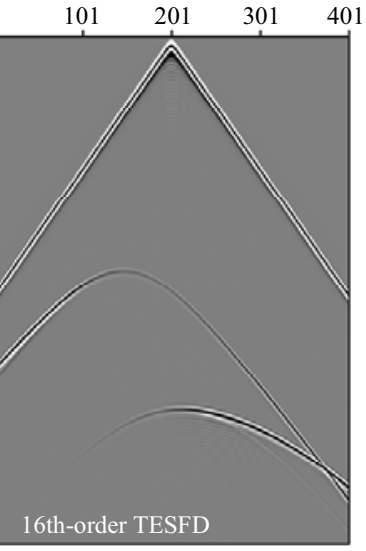

(c)

Fig. 3. Seismograms for the fault model computed by: (a) the 8th-order LSSFD operator, (b) the 8th-order TESFD operator, and (c) the 16th-order TESFD operator, respectively.

Trace Number

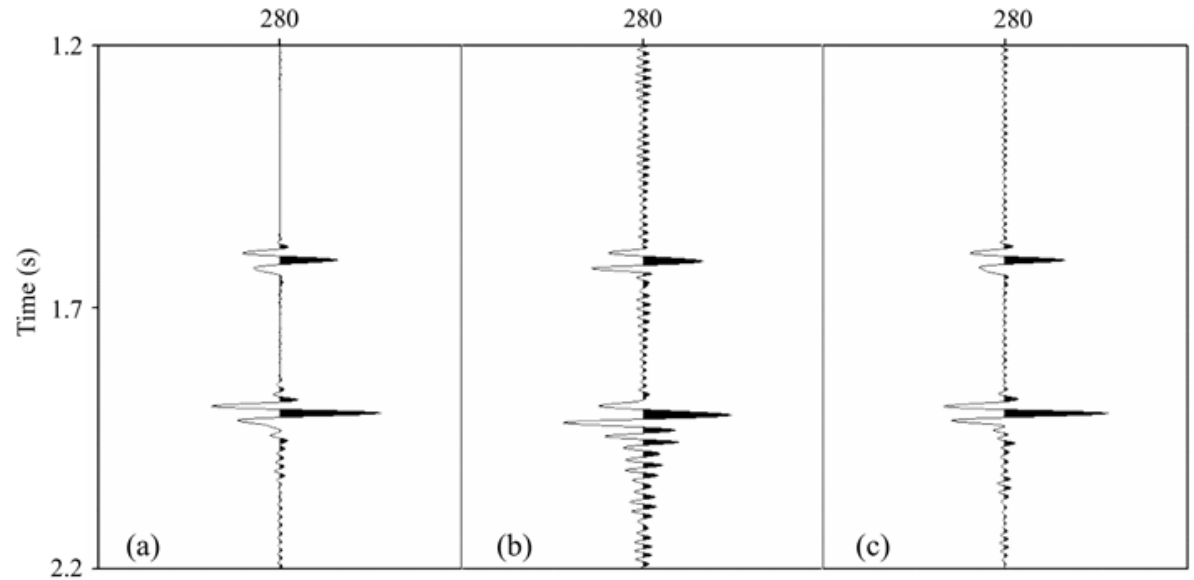

Fig. 4. Comparison of the records extracted from: (a) Fig. 3a, (b) Fig. 3b, and (c) Fig. 3c, respectively. Here, all the records are shown on the same amplitude scales.

Figure 6 shows a comparison of computing costs for different SFD operators with different spatial difference operator length in acoustic modeling. Obviously, the computing costs of the LSSFD operators are very close to that of the TESFD operators under the same spatial difference operator length. 


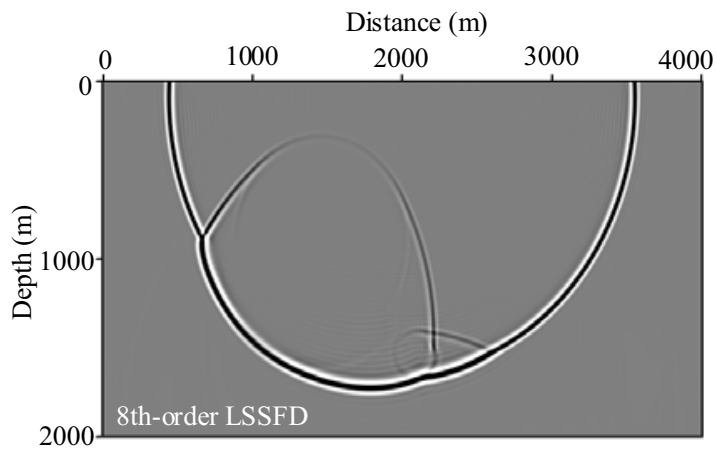

(a)

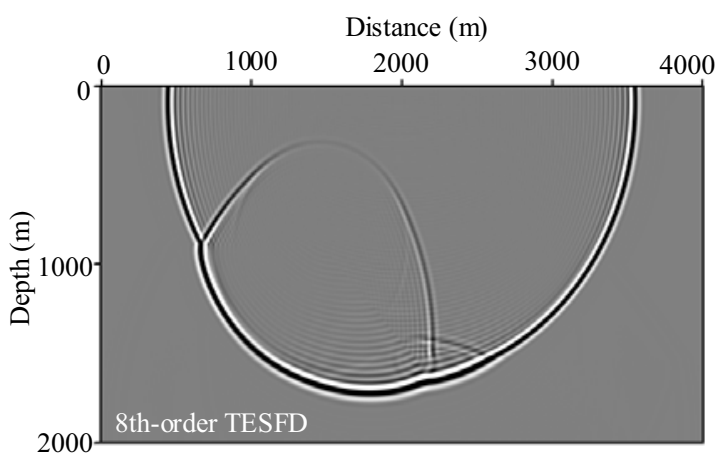

(b)

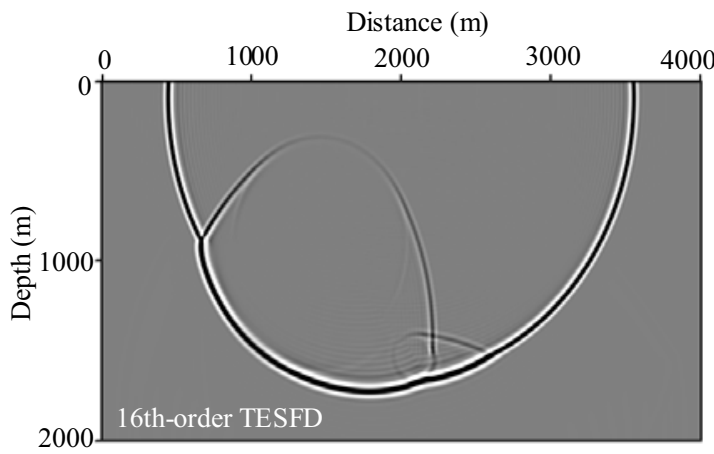

(c)

Fig. 5. Snapshots for the fault model computed by: (a) the 8th-order LSSFD operator, (b) the 8th-order TESFD operator, and (c) the 16th-order TESFD operator, respectively. Here, all the snapshots are shown on the same amplitude scales.

Therefore, the comparison shows that the solving of wave equation using LSSFD operator has hardly any extra computing costs in comparison with that using the TESFD operator. Obviously, the application of the LSSFD operator for RTM has the same conclusion in the computing costs.

Figure 7 shows the final RTM images using the 8th-order LSSFD operator, the 8th-order TESFD operator, and the 16th-order TESFD operator, re- 


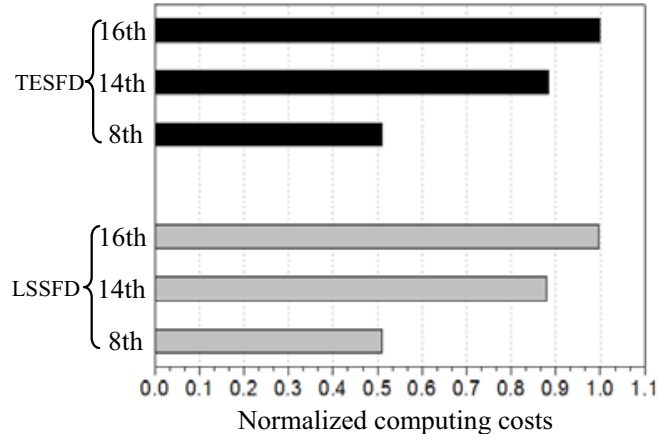

Fig. 6. Comparison of the computing costs for the TESFD and the LSSFD in acoustic modeling. The normalized computing costs of 8th-, 14th-, 16th-order TESFD operators and 8th-, 14th-, 16th-order LSSFD operators are shown in this figure.

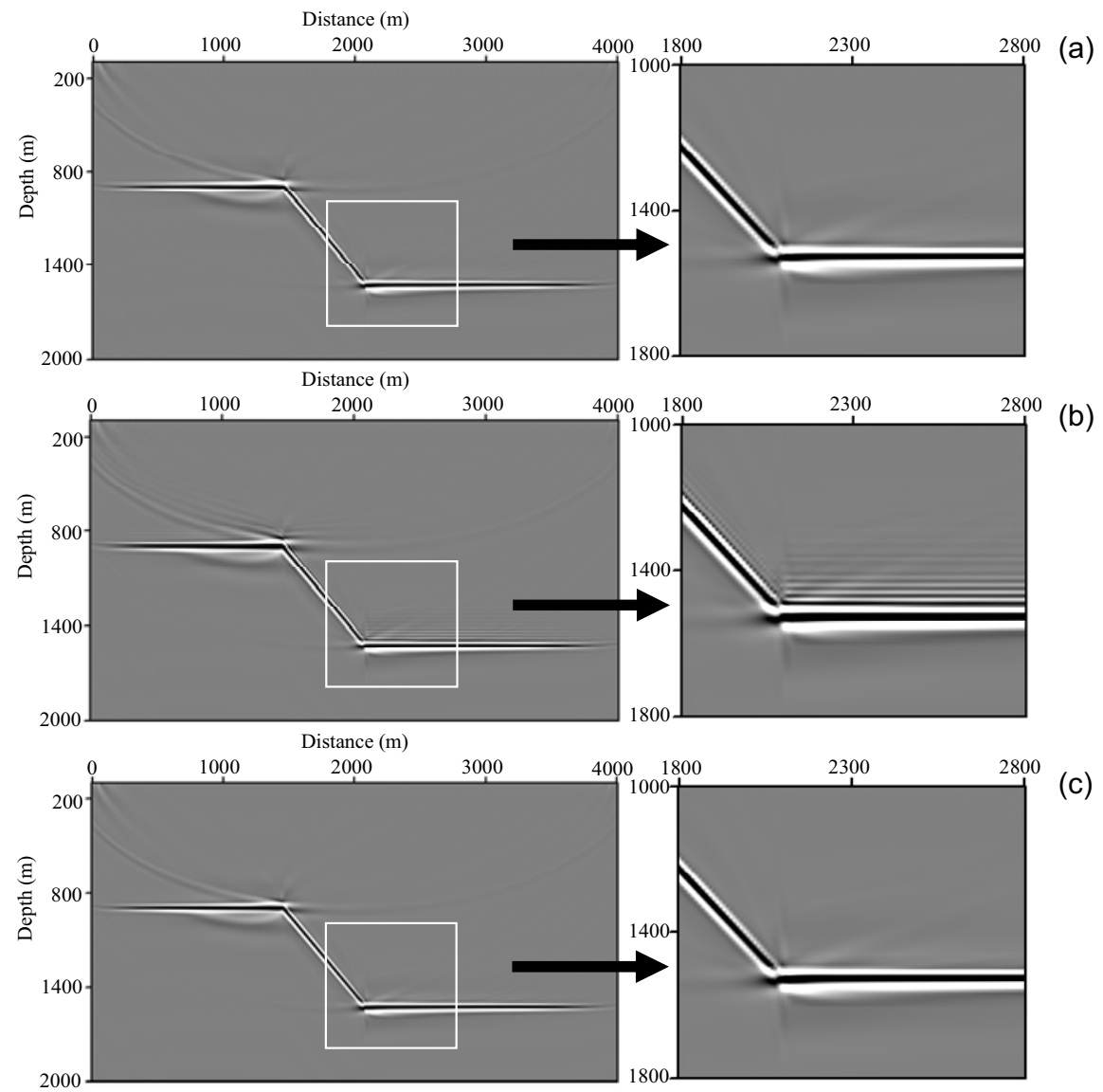

Fig. 7. The RTM images for the fault model by: (a) the 8th-order LSSFD operator, (b) the 8th-order TESFD operator, (c) the 16th-order TESFD operator, respectively. 
spectively. From Fig. 7, we observe that the image using the 8th-order LSSFD operator is very clear and shows the structure of the real model. However, the image using the 8th-order TESFD operator has some artifacts along the reflector interfaces resulting from numerical dispersion effects in wave propagation. So the model test demonstrates the acoustic RTM using the LSSFD operator can obtain the better image than that using the TESFD operator under the same spatial difference operator length. Additionally, the image using the 16th-order TESFD operator is of less dispersion noise and very clear. By comparing Fig. 7a with $\mathrm{c}$, we find that the LSSFD operator can adopt a shorter spatial difference operator in RTM while preserving the high imaging accuracy, compared to the TESFD operator.

\subsection{RTM for salt model}

To further examine the image quality, we test the RTM algorithms on the 2D Society of Exploration Geophysicists/European Association of Geoscientists and Engineers (SEG/EAGE) salt model. The salt model is discretized into 600 (in $x$ ) by 200 (in depth) grid points, with grid intervals of $20 \mathrm{~m}$ (in $x$ ) and $20 \mathrm{~m}$ (in depth). The $P$-wave velocity is illustrated in Fig. $8 \mathrm{a}$, and Fig. $8 \mathrm{~b}$ is

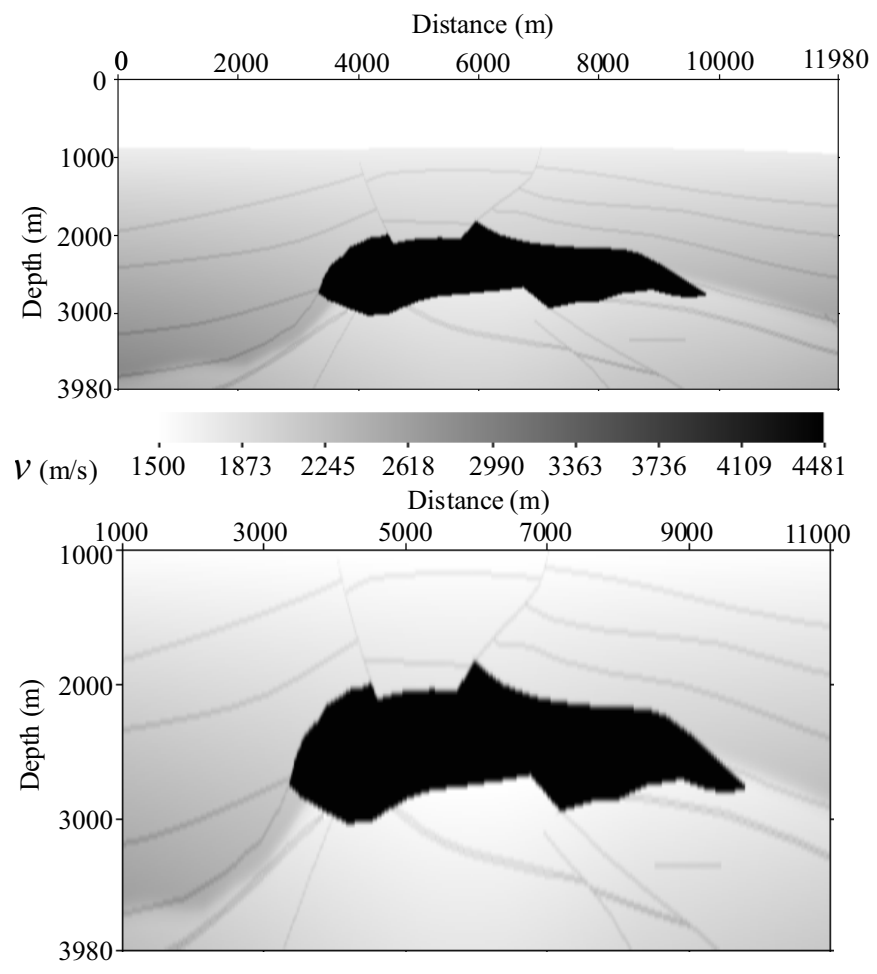

(a)

(b)

Fig. 8: (a) Velocity for the 2D SEG/ EAGE salt model, and (b) zoom of the 2D SEG/EAGE salt model. 
the zoom of Fig. 8a. The density is constant. We use the 8th-order LSSFD to generate synthetic data for 116 sources from location 200 to $11700 \mathrm{~m}$ with a source spacing of $100 \mathrm{~m}$. The source function is a $30 \mathrm{~Hz}$ Ricker wavelet. The total record time is $8 \mathrm{~s}$ and the time step is $0.001 \mathrm{~s}$. We perform the acoustic RTM using the TESFD and the LSSFD operators, respectively. Here, the true model velocity is taken as the migration velocity.

Figure 9 shows the final RTM images by the 6th-order LSSFD and the 6th-order TESFD operators, respectively. The image using the LSSFD operators is very clear and accurate by comparison with the true model (Fig. 8b). However, the image using the TESFD operator has some artifacts along the reflector interfaces and the boundary of the salt body, which result from the numerical dispersion. By comparing the images using two kinds of SFD operators, we observe that the image using the LSSFD operator has better image quality than that using the TESFD operator.

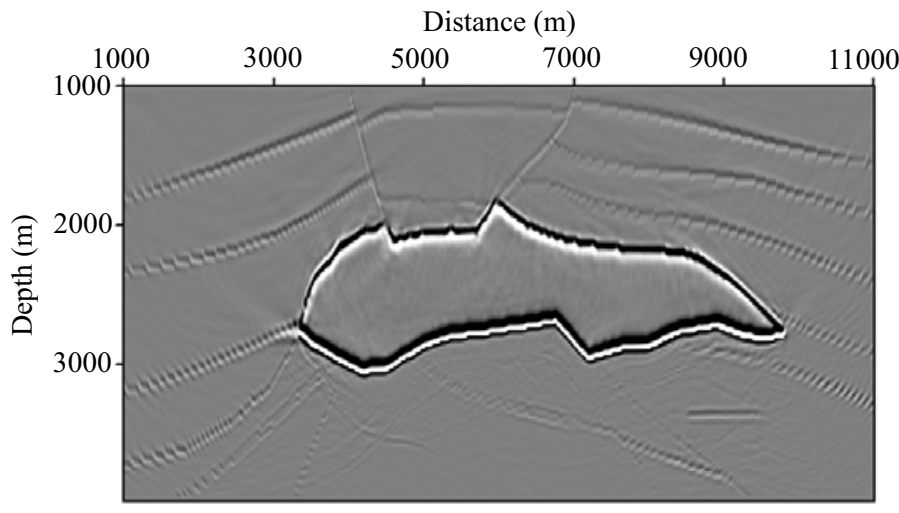

(a)

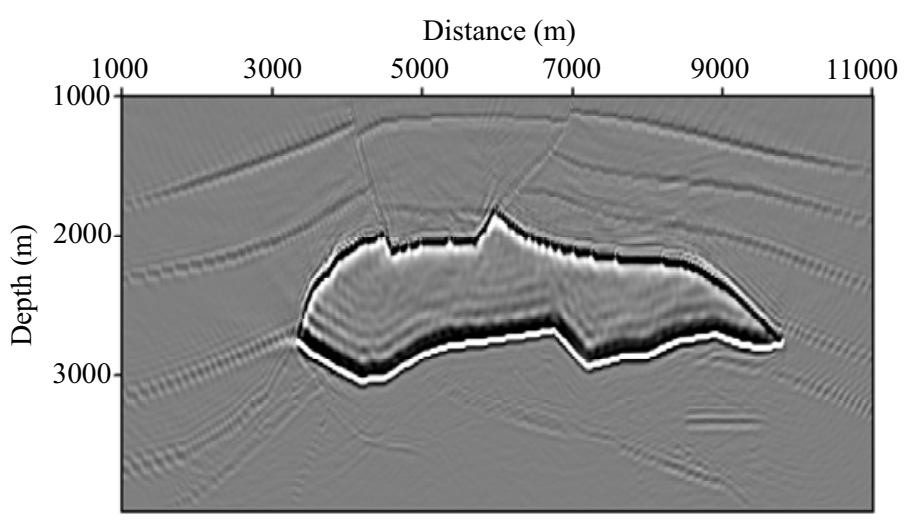

(b)

Fig. 9. The RTM images for the 2D SEG/EAGE salt model by: (a) the LSSFD operator, and (b) the TESFD operator, respectively. 


\section{REAL DATA APPLICATION}

We use a real 2D seismic data from the northeast China to test RTM algorithms based on the LSSFD operator and the TESFD operator under the same spatial difference operator length (8th-order), respectively. The data consists of 100 shot gathers with a source interval of $100 \mathrm{~m}$. For each shot gather, there are 159 traces with a trace interval of $50 \mathrm{~m}$. The time sampling interval for receiver recording is $0.001 \mathrm{~s}$. Figure 10 shows the common-shot gathers. Figure 11 is the migration velocity model for the real data test. Grid

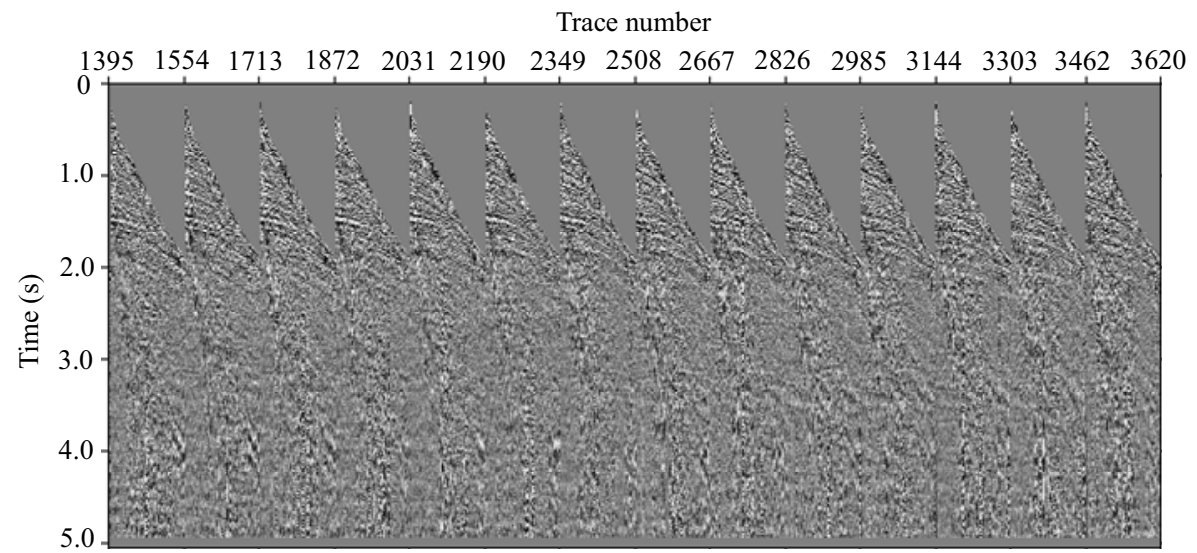

Fig. 10. The common-shot gathers (only fourteen shots are shown).

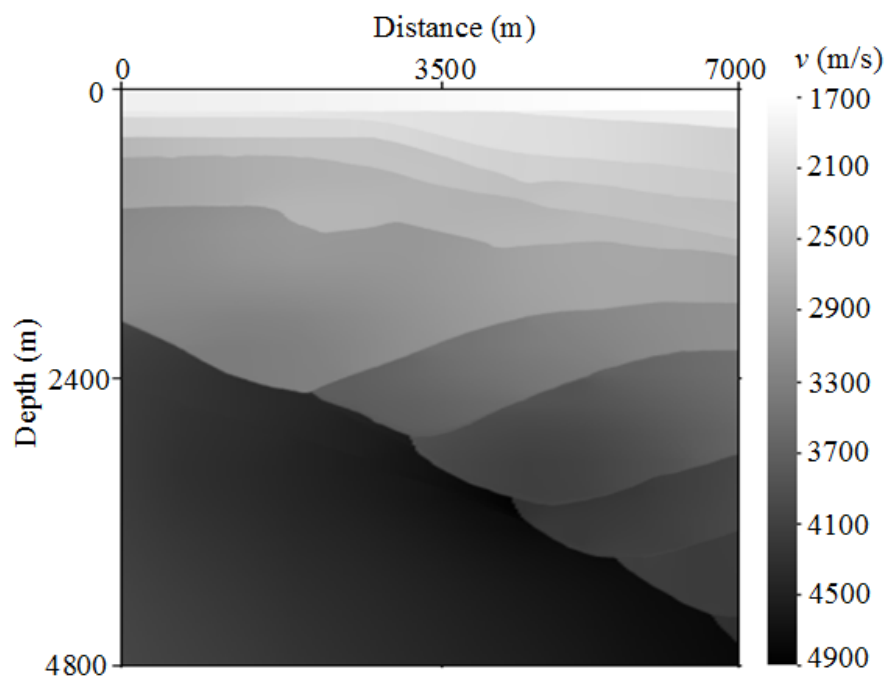

Fig. 11. The migration velocity model for the real data test. 
intervals are $10 \mathrm{~m}$ (in $x$ ) and $10 \mathrm{~m}$ (in $z$ ). The RTM images obtained by the LSSFD operator and the TESFD operator are shown in Fig. 12, respectively. Figure 13 is the partial zoom of RTM sections in Fig. 12 (black boxes). Comparing these two images, the reflectors in the RTM image by the LSSFD operator are characterized by better focusing and balancing of amplitudes, and contain fewer artifacts compared to the RTM image by the TESFD operator. This suggests that the numerical dispersion affects the quality of the RTM image. The real data test shows that the RTM by the LSSFD operator produced a better image than that by the TESFD operator.

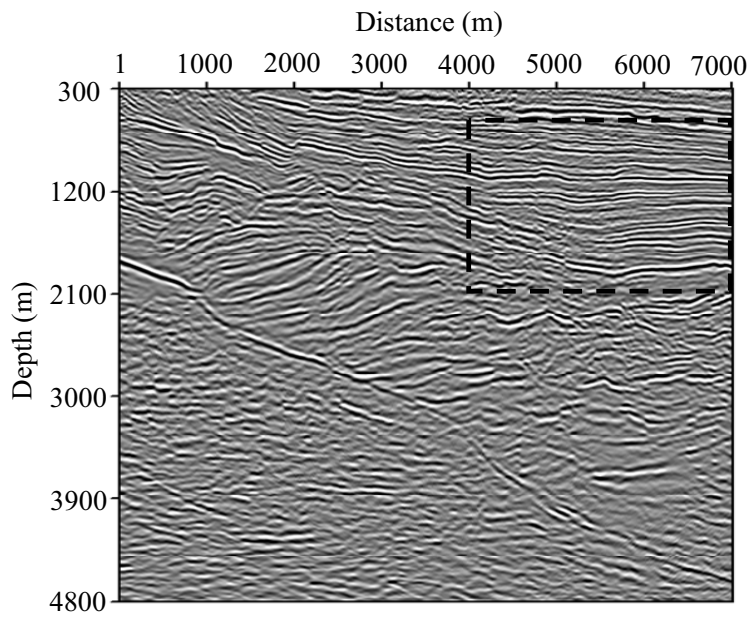

(a)

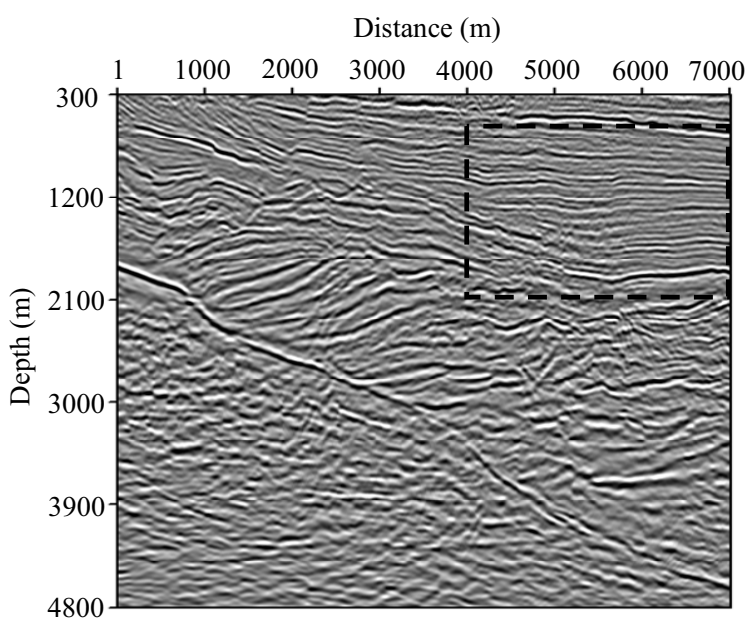

(b)

Fig. 12. The RTM images for the real data obtained by: (a) the LSSFD operator, and (b) the TESFD operator. The black boxes indicate the areas for zoom view. 

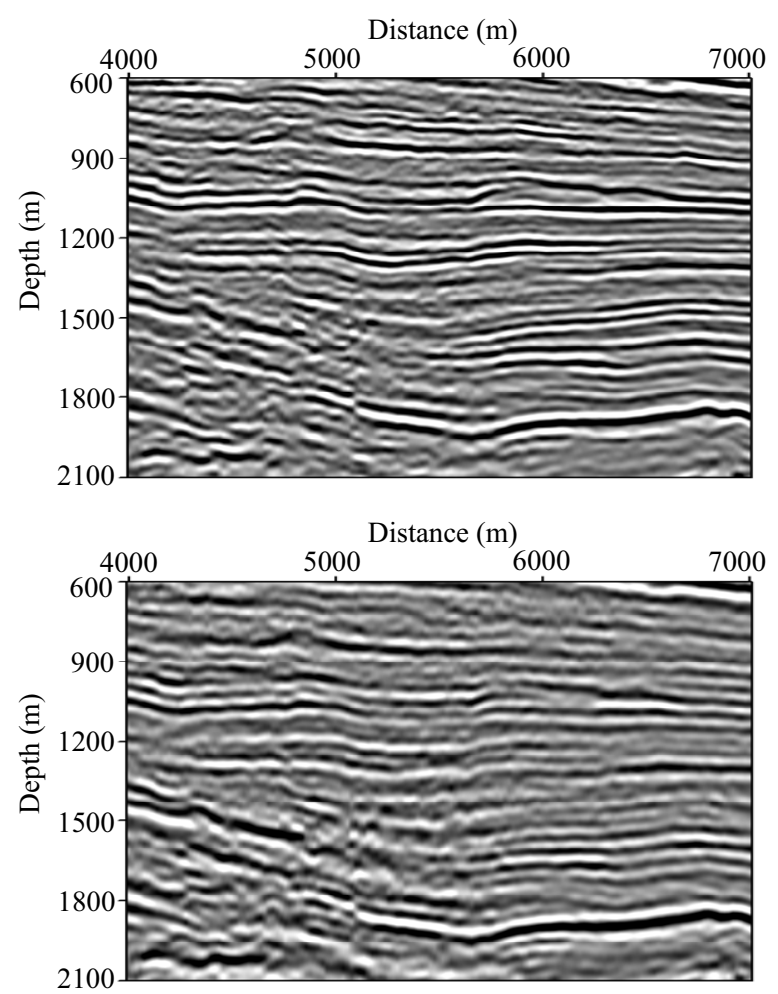

(a)

(b)

Fig. 13. The zoom views of the black boxes in Fig. 12: (a) the RTM image obtained by the LSSFD operator, and (b) the RTM image obtained by the TESFD operator.

\section{CONCLUSIONS}

We propose the LSSFD operator to perform wavefield forward and backward extrapolations for acoustic prestack RTM, and obtain a new antidispersion RTM algorithm that can use short spatial difference operator. The numerical results demonstrate that the LSSFD operator can effectively attenuate the numerical dispersion on the larger frequency zone, and the acoustic modeling accuracy by the LSSFD operators is much higher than that by the TESFD operators under the same spatial difference operator length. Moreover, the synthetic and real data tests demonstrate that the acoustic RTM using the LSSFD operator can effectively improve the image quality comparing with that using TESFD operator without extra computing costs. And the LSSFD method can adopt a shorter spatial difference operator to reduce the computing cost with preserving the modeling and imaging accuracy. Furthermore, the LSSFD operator can be easily extended to other RTM algorithms based on different wave equations. 
Acknowledgements. This research is supported by the China Postdoctoral Science Foundation funded project under contract numbers 2012M520374, 2013T60169, the National High Technology Research and Development Program of China ("863" Program) under contract number 2012AA061202, and China National Petroleum Corporation (the Scientific Research and Technology Development Project).

\section{APPENDIX}

\section{The difference coefficients for the LSSFD operators}

In this Appendix, we derive the difference coefficients for the LSSFD operators in detail, and list the values of the difference coefficients for LSSFD operators and TESFD operators, respectively.

Let

$$
\varphi_{m}(\beta)=\sin [(2 m-1) \beta] .
$$

So Eq. 5 can be rewritten as

$$
E\left(c_{1}, c_{2}, \cdots c_{M}\right)=\int_{0}^{b}\left\{\beta-\sum_{m=1}^{M} c_{m} \varphi_{m}(\beta)\right\}^{2} d \beta .
$$

Let

$$
\begin{gathered}
\left(\varphi_{n}, \varphi_{m}\right)=\int_{0}^{b} \varphi_{n}(\beta) \varphi_{m}(\beta) d \beta, \\
\left(\beta, \varphi_{m}\right)=\int_{0}^{b} \beta \varphi_{m}(\beta) d \beta,
\end{gathered}
$$

where $n, m=1,2, \ldots, M$.

Substituting Eq. A2 into Eq. 6, and simplifying them by introducing Eqs. A3 and A4, we finally obtain

$$
\left[\begin{array}{cccc}
\left(\varphi_{1}, \varphi_{1}\right) & \left(\varphi_{1}, \varphi_{2}\right) & \cdots & \left(\varphi_{1}, \varphi_{M}\right) \\
\left(\varphi_{2}, \varphi_{1}\right) & \left(\varphi_{2}, \varphi_{2}\right) & \cdots & \left(\varphi_{2}, \varphi_{M}\right) \\
\vdots & & & \vdots \\
\left(\varphi_{M}, \varphi_{1}\right) & \left(\varphi_{M}, f_{2}\right) & \cdots & \left(\varphi_{M}, \varphi_{M}\right)
\end{array}\right]\left[\begin{array}{c}
c_{1} \\
c_{2} \\
\vdots \\
c_{M}
\end{array}\right]=\left[\begin{array}{c}
\left(\beta, \varphi_{1}\right) \\
\left(\beta, \varphi_{2}\right) \\
\vdots \\
\left(\beta, \varphi_{M}\right)
\end{array}\right] .
$$


We use Gaussian elimination to solve linear Eq. A5 for obtaining the difference coefficients for LSSFD operators (Yang et al. 2014), then apply the LSSFD operators to solve wave equation and perform RTM.

Tables A1 and A2 list the 4th, 6th, 8th, 10th, 12th, 14th, 16th, 18th, 20th, and 22nd-order SFD coefficients calculated by the Taylor-series expansion method and the least squares method, respectively.

Table A 1

Difference coefficients of the TESFD operators (Liu and Sen 2009)

\begin{tabular}{|c|c|c|c|c|c|}
\hline$c_{m}$ & $M=2$ & $M=3$ & $M=4$ & $M=5$ & $M=6$ \\
\hline$c_{1}$ & $1.125000 \mathrm{e}+0$ & $1.171875 \mathrm{e}+0$ & $1.196289 \mathrm{e}+0$ & $1.211243 \mathrm{e}+0$ & $1.221336 \mathrm{e}+0$ \\
$c_{2}$ & $-4.166667 \mathrm{e}-2$ & $-6.510417 \mathrm{e}-2$ & $-7.975260 \mathrm{e}-2$ & $-8.972168 \mathrm{e}-2$ & $-9.693146 \mathrm{e}-2$ \\
$c_{3}$ & & $4.687500 \mathrm{e}-2$ & $9.570313 \mathrm{e}-3$ & $1.384277 \mathrm{e}-2$ & $1.744766 \mathrm{e}-2$ \\
$c_{4}$ & & & $-6.975446 \mathrm{e}-4$ & $-1.765660 \mathrm{e}-3$ & $-2.967290 \mathrm{e}-3$ \\
$c_{5}$ & & & & $1.186795 \mathrm{e}-4$ & $3.590054 \mathrm{e}-4$ \\
$c_{6}$ & & & & & $-2.184781 \mathrm{e}-5$ \\
\hline$c_{m}$ & $M=7$ & $M=8$ & $M=9$ & $M=10$ & $M=11$ \\
\hline$c_{1}$ & $1.228606 \mathrm{e}+0$ & $1.234091 \mathrm{e}+0$ & $1.238376 \mathrm{e}+0$ & $1.241816 \mathrm{e}+0$ & $1.244638 \mathrm{e}+0$ \\
$c_{2}$ & $-1.023839 \mathrm{e}-1$ & $-1.066498 \mathrm{e}-1$ & $-1.100779 \mathrm{e}-1$ & $-1.128924 \mathrm{e}-1$ & $-1.152443 \mathrm{e}-1$ \\
$c_{3}$ & $2.047677 \mathrm{e}-2$ & $2.303637 \mathrm{e}-2$ & $2.521784 \mathrm{e}-2$ & $2.709417 \mathrm{e}-2$ & $2.872242 \mathrm{e}-2$ \\
$c_{4}$ & $-4.178933 \mathrm{e}-3$ & $-5.342386 \mathrm{e}-3$ & $-6.433123 \mathrm{e}-3$ & $-7.443453 \mathrm{e}-3$ & $-8.373884 \mathrm{e}-3$ \\
$c_{5}$ & $6.894535 \mathrm{e}-4$ & $1.077271 \mathrm{e}-3$ & $1.496785 \mathrm{e}-3$ & $1.929784 \mathrm{e}-3$ & $2.363985 \mathrm{e}-3$ \\
$c_{6}$ & $-7.692250 \mathrm{e}-5$ & $-1.664189 \mathrm{e}-4$ & $-2.862801 \mathrm{e}-4$ & $-4.306130 \mathrm{e}-4$ & $-5.934385 \mathrm{e}-4$ \\
$c_{7}$ & $4.236515 \mathrm{e}-6$ & $1.702171 \mathrm{e}-5$ & $4.099395 \mathrm{e}-5$ & $7.707717 \mathrm{e}-5$ & $1.249670 \mathrm{e}-4$ \\
$c_{8}$ & & $-8.523464 \mathrm{e}-7$ & $-3.848877 \mathrm{e}-6$ & $-1.021650 \mathrm{e}-5$ & $-2.085870 \mathrm{e}-5$ \\
$c_{9}$ & & & $1.762665 \mathrm{e}-7$ & $8.837806 \mathrm{e}-7$ & $2.564127 \mathrm{e}-6$ \\
$c_{10}$ & & & & $-3.723759 \mathrm{e}-8$ & $-2.052722 \mathrm{e}-7$ \\
$c_{11}$ & & & & & $8.001648 \mathrm{e}-9$ \\
\hline
\end{tabular}


Difference coefficients of the LSSFD operators $(b=1.02)$

\begin{tabular}{|l|r|r|r|r|r|}
\hline$c_{m}$ & $M=2$ & $M=3$ & $M=4$ & $M=5$ & $M=6$ \\
\hline$c_{1}$ & $1.188401 \mathrm{e}+0$ & $1.216624 \mathrm{e}+0$ & $1.230862 \mathrm{e}+0$ & $1.239407 \mathrm{e}+0$ & $1.245095 \mathrm{e}+0$ \\
$c_{2}$ & $-7.046382 \mathrm{e}-2$ & $-9.197724 \mathrm{e}-2$ & $-1.034123 \mathrm{e}-1$ & $-1.105315 \mathrm{e}-1$ & $-1.153979 \mathrm{e}-1$ \\
$c_{3}$ & & $1.300041 \mathrm{e}-2$ & $2.011671 \mathrm{e}-2$ & $2.496329 \mathrm{e}-2$ & $2.848442 \mathrm{e}-2$ \\
$c_{4}$ & & & $-3.245760 \mathrm{e}-3$ & $-5.804879 \mathrm{e}-3$ & $-7.899473 \mathrm{e}-3$ \\
$c_{5}$ & & & & $9.358680 \mathrm{e}-4$ & $1.898222 \mathrm{e}-3$ \\
$c_{6}$ & & & & & $-2.935304 \mathrm{e}-4$ \\
\hline$c_{m}$ & $M=7$ & $M=8$ & $M=9$ & $M=10$ & $M=11$ \\
\hline$c_{1}$ & $1.249150 \mathrm{e}+0$ & $1.252186 \mathrm{e}+0$ & $1.254544 \mathrm{e}+0$ & $1.256428 \mathrm{e}+0$ & $1.257967 \mathrm{e}+0$ \\
$c_{2}$ & $-1.189375 \mathrm{e}-1$ & $-1.216289 \mathrm{e}-1$ & $-1.237449 \mathrm{e}-1$ & $-1.254524 \mathrm{e}-1$ & $-1.268598 \mathrm{e}-1$ \\
$c_{3}$ & $3.116091 \mathrm{e}-2$ & $3.326513 \mathrm{e}-2$ & $3.496337 \mathrm{e}-2$ & $3.636307 \mathrm{e}-2$ & $3.753689 \mathrm{e}-2$ \\
$c_{4}$ & $-9.623004 \mathrm{e}-3$ & $-1.105777 \mathrm{e}-2$ & $-1.226716 \mathrm{e}-2$ & $-1.329873 \mathrm{e}-2$ & $-1.418823 \mathrm{e}-2$ \\
$c_{5}$ & $2.812069 \mathrm{e}-3$ & $3.647865 \mathrm{e}-3$ & $4.401731 \mathrm{e}-3$ & $5.078779 \mathrm{e}-3$ & $5.686916 \mathrm{e}-3$ \\
$c_{6}$ & $-6.655106 \mathrm{e}-4$ & $-1.066144 \mathrm{e}-3$ & $-1.468116 \mathrm{e}-3$ & $-1.857840 \mathrm{e}-3$ & $-2.228932 \mathrm{e}-3$ \\
$c_{7}$ & $9.728191 \mathrm{e}-5$ & $2.437491 \mathrm{e}-4$ & $4.198223 \mathrm{e}-4$ & $6.117512 \mathrm{e}-4$ & $8.105434 \mathrm{e}-4$ \\
$c_{8}$ & & $-3.351959 \mathrm{e}-5$ & $-9.196027 \mathrm{e}-5$ & $-1.694350 \mathrm{e}-4$ & $-2.605010 \mathrm{e}-4$ \\
$c_{9}$ & & & $1.188808 \mathrm{e}-5$ & $3.543912 \mathrm{e}-5$ & $6.954928 \mathrm{e}-5$ \\
$c_{10}$ & & & & $-4.311393 \mathrm{e}-6$ & $-1.387702 \mathrm{e}-5$ \\
$c_{11}$ & & & & & $1.591802 \mathrm{e}-6$ \\
\hline
\end{tabular}

References

Baysal, E., D.D. Kosloff, and J.W.C. Sherwood (1983), Reverse time migration, Geophysics 48, 11, 1514-1524, DOI: 10.1190/1.1441434.

Chattopadhyay, S., and G.A. McMechan (2008), Imaging conditions for prestack reverse-time migration, Geophysics 73, 3, S81-S89, DOI: 10.1190/ 1.2903822.

Chu, C., and P.L. Stoffa (2012), Determination of finite-difference weights using scaled binomial windows, Geophysics 77, 3, W17-W26, DOI: 10.1190/ geo2011-0336.1.

Claerbout, J.F. (1985), Imaging the Earth's Interior, Blackwell Scientific Publs., Oxford. 
Dong, L.G., Z.T. Ma, J.Z. Cao, H.Z. Wang, J.H. Gao, B. Lei, and S.Y. Xu (2000), A staggered-grid high-order difference method of one-order elastic wave equation, Chin. J. Geophys. 43, 3, 411-419 (in Chinese).

Igel, H., B. Riollet, and P. Mora (1992), Accuracy of staggered 3-D finite-difference grids for anisotropic wave propagation. In: 62th SEG Ann. Meeting, 25-29 October 1992, New Orleans, USA, SEG-1992-1244, Society of Exploration Geophysicists, 1244-1246.

Kindelan, M., A. Kamel, and P. Sguazzero (1990), On the construction and efficiency of staggered numerical differentiators for the wave equation, Geophysics 55, 1, 107-110, DOI: 10.1190/1.1442763.

Kosloff, D., R.C. Pestana, and H. Tal-Ezer (2010), Acoustic and elastic numerical wave simulations by recursive spatial derivative operators, Geophysics $\mathbf{7 5}$, 6, T167-T174, DOI: 10.1190/1.3485217.

Leveille, J.P., I.F. Jones, Z.-Z. Zhou, B. Wang, and F. Liu (2011), Subsalt imaging for exploration, production, and development: A review, Geophysics 76, 5, WB3-WB20, DOI: 10.1190/geo2011-0156.1.

Li, J., D. Yang, and F. Liu (2013), An efficient reverse time migration method using local nearly analytic discrete operator, Geophysics 78, 1, S15-S23, DOI: 10.1190/geo2012-0247.1.

Liu, F., S.A. Morton, J.P. Leveille, and G. Zhang (2008), An anti-dispersion wave equation for modeling and reverse-time migration. In: 78th SEG Ann. Meeting, 9-14 November 2008, Las Vegas, USA, SEG-2008-2277, Society of Exploration Geophysicists, 2277-2281.

Liu, F., G. Zhang, S.A. Morton, and J.P. Leveille (2011), An effective imaging condition for reverse-time migration using wavefield decomposition, Geophysics 76, 1, S29-S39, DOI: 10.1190/1.3533914.

Liu, Y. (2013), Globally optimal finite-difference schemes based on least squares, Geophysics 78, 4, T113-T132, DOI: 10.1190/geo2012-0480.1.

Liu, Y., and M.K. Sen (2009), An implicit staggered-grid finite-difference method for seismic modelling, Geophys. J. Int. 179, 1, 459-474, DOI: 10.1111/ j.1365-246X.2009.04305.x.

Liu, Y., and M.K. Sen (2011), Scalar wave equation modeling with time-space domain dispersion-relation-based staggered-grid finite-difference schemes, Bull. Seismol. Soc. Am. 101, 1, 141-159, DOI: 10.1785/0120100041.

Liu, Y., C. Li, and Y. Mou (1998), Finite-difference numerical modeling of any even-order accuracy, Oil Geophys. Prospect. 33, 1-10 (in Chinese).

McMechan, G.A. (1983), Migration by extrapolation of time-dependent boundary values, Geophys. Prospect. 31, 3, 413-420, DOI: 10.1111/j.13652478.1983.tb01060.x.

Pei, Z. (2004), Numerical modeling using staggered-grid high order finite difference of elastic wave equation on arbitrary relief surface, Oil Geophys. Prospect. 39, 629-634 (in Chinese). 
Pestana, R.C., and P.L. Stoffa (2009), Rapid expansion method (REM) for timestepping in reverse time migration (RTM). In: 79th SEG Ann. Meeting, 2530 October 2009, Houston, USA, SEG-2009-2819, Society of Exploration Geophysicists, 2819-2823.

Pestana, R.C., and P.L. Stoffa (2010), Time evolution of the wave equation using rapid expansion method, Geophysics 75, 4, T121-T131, DOI: 10.1190/ 1.3449091 .

Pestana, R.C., B. Ursin, and P.L. Stoffa (2012), Rapid expansion and pseudo spectral implementation for reverse time migration in VTI media, J. Geophys. Eng. 9, 3, 291, DOI: 10.1088/1742-2132/9/3/291.

Tessmer, E. (2011), Using the rapid expansion method for accurate time-stepping in modeling and reverse-time migration, Geophysics 76, 4, S177-S185, DOI: 10.1190/1.3587217.

Virieux, J. (1986), P-SV wave propagation in heterogeneous media: Velocity-stress finite-difference method, Geophysics 51, 4, 889-901, DOI: 10.1190/ 1.1442147.

Yan, H., and Y. Liu (2013), Visco-acoustic prestack reverse-time migration based on the time-space domain adaptive high-order finite-difference method, Geophys. Prospect. 61, 5, 941-954, DOI: 10.1111/1365-2478.12046.

Yan, H., Y. Liu, and H. Zhang (2013), Prestack reverse-time migration with a timespace domain adaptive high-order staggered-grid finite-difference method, Explor. Geophys. 44, 2, 77-86, DOI: 10.1071/EG12047.

Yang, L., H. Yan, and H. Liu (2014), Least squares staggered-grid finite-difference for elastic wave modelling, Explor. Geophys. 45, 4, 255-260, DOI: 10.1071/EG13087.

Zhang, J.-H., and Z.-X. Yao (2013), Optimized finite-difference operator for broadband seismic wave modeling, Geophysics 78, 1, A13-A18, DOI: 10.1190/geo2012-0277.1.

Zhang, Y., and J. Sun (2009), Practical issues of reverse time migration: True amplitude gathers, noise removal and harmonic-source encoding, First Break 26, 19-25.

Zhang, Y., and G. Zhang (2009), One-step extrapolation method for reverse time migration, Geophysics 74, 4, A29-A33, DOI: 10.1190/1.3123476.

Zhang, Y., P. Zhang, and H. Zhang (2010), Compensating for visco-acoustic effects in reverse-time migration. In: 80th SEG Ann. Meeting, 17-22 October 2010, Denver, USA, SEG-2010-3160, Society of Exploration Geophysicists, 3160-3164.

Received 19 January 2014

Received in revised form 18 April 2014 